\title{
DERECHOS FUNDAMENTALES: INDETERMINACIÓN CONCEPTUAL, PLASMACIÓN JURÍDICA E INTERPRETACIÓN*
}

\author{
Fundamental Rights: conceptual indeterminacy, \\ legal embodiment and interpretation
}

\author{
Estela Camus García \\ Universidad Loyola Andalucía (España) \\ escamus@uloyola.es
}

http://dx.doi.org/10.18543/ed-66(2)-2018pp105-115

Recibido: 30.10 .2018

Aceptado: 21.11.2018

\section{Resumen}

Este trabajo presenta una reflexión en torno al papel del Legislador y su competencia para la decisión. Fundamentalmente, propongo un debate sobre las posibles colisiones entre conceptos a partir de los que replantear las relaciones entre órganos constitucionales, la teoría de la Constitución, la teoría del Estado y la teoría de la democracia. En estas tensiones pueden vislumbrarse matices diferenciados entre dos estadios: el de la creación del Derecho y el de la aplicación del mismo.

\section{Palabras clave}

Derechos Fundamentales; interpretación; indeterminación conceptual; teoría de la democracia; teoría de la Constitución.

* Cómo citar / Citation 'Chicago-Deusto' (Autor-fecha / Author-date / Lista de referencias / Reference list entries): Camus García, Estela. 2018. «Derechos fundamentales: indeterminación conceptual, plasmación jurídica e interpretación». Estudios de Deusto 66, n. ${ }^{\circ}$ 2: 105-115. http://dx.doi.org/10.18543/ed-66(2)-2018pp105-115. 


\section{Abstract}

This paper presents a reflection on the role of the Legislator and its competence for the decision. I propose a debate about the range of possible collisions between concepts from which to reconsider the relations between constitutional organs, the theory of the Constitution, the theory of the State and the theory of democracy. In these tensions can be glimpsed nuances differentiated between two stages: the moment of the Law' creation and those of the Law' application.

\section{Keywords}

Fundamental Rights; interpretation; conceptual indeterminacy; Theory of democracy; Theory of Constitution. 
En el ámbito de la teoría general del Derecho aparece la interpretación jurídica como tema clave y referencial, y sin embargo no es sólo en el hecho de interpretar donde reside la complejidad «sino también [en] la determinación de a quién y con qué alcance corresponde realizar esa actividad. La interpretación es [...] un asunto que tiene mucho que ver también con la organización de los poderes del Estado y con la relación entre ellos, o sea, con su posición constitucional» (Villaverde et alt. 2004: 57) ${ }^{1}$.

Si nos referimos además a la interpretación de normas constitucionales que reconocen derechos fundamentales tomamos contacto con la indeterminación -que en ciertos casos podría tratarse de una aproximación a la idea de inconmensurabilidad misma- y con la no adecuación al esquema condicional y teóricamente cerrado de los enunciados jurídicos clásicos (Villaverde et alt. 2004:57; Schlag, 2009: 35-62; Greco: 2006: 1-22)2.

Respecto a la indeterminación encontramos en el Estudio Introductorio a la Teoría de los derechos fundamentales de Alexy una afirmación que nos sirve de inicio al debate sobre la competencia del Legislador:

«Una parte importante de la teoría de los principios es la dogmática de los márgenes de acción. Mediante los márgenes de acción estructural del Legislador, es decir, el margen de acción para la determinación de fines y el margen de acción para la elección de los medios, la teoría de los principios garantiza el respeto del principio democrático. De esta manera, se reconoce que la legislación no constituye una mera aplicación de los mandatos de optimización. Las disposiciones de derecho fundamentales no ordenan ni prohíben nada en relación con muchos casos posibles. Pues bien, en donde la Constitución nada prescribe, el Legislador tiene competencia para decidir» ${ }^{3}$.

1 Villaverde, Ignacio; Requejo, Paloma; Aláez, Benito; Fernández, Ignacio; Bastida, Fco. José; Presno, Miguel A. Teoría general de los derechos fundamentales en la Constitución española de 1978. Madrid, Tecnos, 2004, p. 57. Véase también: Villaverde, Ignacio. «Verdad y Constitución. Una incipiente dogmática de las ficciones constitucionales», en Revista española de derecho constitucional. Año N. 36, Núm. 106, 2016, págs: 149201; Fioravanti, M. Constitucionalismo. Experiencias históricas y tendencias actuales. Madrid, Trotta 2014. 167 p. y Massini Correas, Carlos Ignacio. «La interpretación jurídica como interpretación práctica», en Persona y derechos: Revista de fundamentación de las Instituciones Jurídicas y de Derechos Humanos, N. 52, 20015, págs: 413-444

2 Íbidem; Schlag, Pierre. «The dedifferentiation problem», en Continental Philosophy Review, 200942 (1). Págs.: 35-62. Un ejemplo muy ilustrativo en al ámbito de la salud se encuentra en: Greco, M. «The Politics of Indeterminacy and the Right to Health», en Theory, Culture and Society, 2006, 21. 6, págs: 1-22 Véase también: Garcia Amado, J. A. Teorías del sistema jurídico y concepto de derecho. Anuario de Filosofía del Derecho, 2, 1985, págs. 297316.

3 Bernal Pulido, Carlos. «Estudio Introductorio», en Alexy, Robert. Teoría de los derechos fundamentales. Madrid, Centro de Estudios Constitucionales, 1993, LII-LIII. 
Al profundizar en el papel del Legislador y su competencia para la decisión respecto a colisiones entre conceptos desde los que replantear las relaciones entre órganos constitucionales, teoría de la Constitución, teoría del Estado y teoría de la democracia se vislumbran matices entre dos momentos diferenciados y fundamentales: el de la creación del derecho y el de la aplicación del mismo.

En la búsqueda por aclarar parte de la opacidad de los espacios entre ambos momentos -el de creación del derecho y el de la aplicación del mismo- considero valiosas, y muy apropiadas para un enlace, a su vez, con el debate actual sobre los no-ciudadanos, los sujetos de derechos y la titularidad de éstos, una serie de aportaciones. En primer lugar reflexiones como la desarrollada por Robert Alexy respecto a la estructura de las normas de derecho fundamental (Alexy, 1989, 1993, 1999) ${ }^{4}$ sumada a la definición del fundamento en el esquema de aproximación a los Derechos Fundamentales en su función subjetiva que lleva a cabo María del Carmen Barranco en La teoría jurídica de los derechos fundamentales (Barranco, 2000) ${ }^{5}$.

También resultan de ayuda para la iniciación al debate las lecturas alrededor del concepto de derecho fundamental y la presentación de los modelos históricos de éstos realizadas por autores como Villaverde, Requejo, Aláez, Fernández, Bastida y Presno en la obra Teoría general de los derechos fundamentales en la Constitución española de 1978 (Villaverde et alt., 2004).

Las preguntas y los cuestionamientos que surgen del acercamiento a la polémica crítica de Anna Pintore sobre los derechos insaciables encarnados en las teorías de Ferrajoli, especialmente a través de su Derecho y razón

De hecho, Bernal continúa más adelante y profundiza sobre la capacidad para decidir:

"De acuerdo con el principio democrático, está permitido al Legislador decidir allí en donde no puede conocerse qué es aquello que los derechos fundamentales ordenan o prohíben. De manera análoga, según el principio del Estado de Derecho, la jurisdicción ordinaria puede decidir allí en donde no puede conocerse si los derechos fundamentales ordenan o prohíben algo en relación con la interpretación del derecho ordinario o la valoración de las pruebas», Ibídem, LV.

${ }^{4}$ Alexy, Robert. Teoría de los derechos fundamentales. Madrid, Centro de Estudios Constitucionales, 1993, 607 p. Véanse del mismo autor: Alexy, Robert. «Constitutional Rights, Balancing, and Rationality», en Ratio Juris. Vol. 16 No. 2 June 2003 Págs. 13140; Law and Correctness. en Legal Theory at the End of the Millennium. Ed. M. D. A. Freeman,. Oxford: Oxford University Press. 1998, págs. 205-221

5 Barranco Avilés, M. del Carmen. La teoría jurídica de los derechos fundamentales. Madrid, Dykinson, 2000, 444 p. Véase también: Díez-Picazo, Luis M. Sistema de Derechos Fundamentales, Madrid, S.L. Civitas Ediciones 2003, 510 p.

${ }_{6}$ Villaverde, Ignacio; Requejo, Paloma; Aláez, Benito; Fernández, Ignacio; Bastida, Fco. José; Presno, Miguel A. Teoría general de los derechos fundamentales en la Constitución española de 1978. Madrid, Tecnos, 2004, 235 p. 
(Pintore, 2001) ${ }^{7}$, también son valiosos en una primera aproximación a la indeterminación conceptual sobre los Derechos Fundamentales a principios del siglo XXI.

El origen de las colisiones que se dan entre principios en el imaginario propuesto por Alexy se matizaría a ojos de Bernal, y de forma paradójica, por una controversia: una idea relacionada con el límite semántico que ofrece la Constitución ${ }^{8}$ y, a la vez, la ampliación misma de significado, de la definición:

«Ahora bien, para garantizar el respeto de los márgenes de acción del Legislador y de la jurisdicción ordinaria, y en consecuencia, dejar sin fundamento las objeciones que acusan a la teoría de los principios de desconocer estos márgenes, es necesario ampliar la definición de los principios en cuanto mandatos de optimización [...] debe ampliarse la definición de los principios de la siguiente manera: Los principios son mandatos de optimización que ordenan que algo sea realizado en la mayor medida posible, de acuerdo con las posibilidades jurídicas, fácticas, y-aquí viene la modificación-epistémicas existentes»?.

Avanzar desde aquí hacia el peso abstracto de los principios en relación a las premisas normativas nos traslada a la complejidad de la sociedad pluralista en la que el juez debe decidir entre varias opciones de acuerdo con el principio democrático (Bernal, 1993: LV) ${ }^{10}$. En un espacio en el cual no puede recurrirse a definiciones cerradas para la aplicación de los principios, no hay soluciones únicas sino deliberación:

7 Pintore, Anna. «Derechos insaciables», en Ferrajoli, Luigi. Los fundamentos de los derechos fundamentales. Madrid, Trotta, 2001, pp: 243-265; Ferrajoli, Luigi. Teoría del garantismo penal. Prólogo de N. Bobbio, Trotta $10^{\text {a }}$ ed., Madrid, 2011, 1024 p. Véanse también: Pintore, Anna. El Derecho sin verdad. Madrid. Dykinson 2006, 234 p.

8 «A pesar de los problemas interpretativos que se originan a partir de los derechos fundamentales, sí resulta meridiano que este margen semántico tiene cierto límites -los límites del significado posible del tener literal de la Constitución-, a los que se suman los que derivan de los límites directamente constitucionales», Bernal Pulido, Carlos. «Estudio Introductorio», en Alexy, Robert. Teoría de los derechos fundamentales. Madrid, Centro de Estudios Constitucionales, 1993, LII; Alexy, Robert. Op. cit. pp-277-279.

9 Bernal Pulido, Carlos. Op. cit. LIII

${ }^{10}$ Así introduce Bernal la idea de Alexy: «[E]stá permitido al Legislador decidir allí en donde no puede conocerse qué es aquello que los derechos fundamentales ordenan o prohíben. De manera análoga, según el principio del Estado de Derecho, la jurisdicción ordinaria puede decidir allí en donde no puede conocerse si los derechos fundamentales ordenan o prohíben algo en relación con la interpretación del derecho ordinario o la valoración de las pruebas», Ibídem LV. 
«en el ámbito de los significados implícitos en los derechos fundamentales, allí en donde no es claro si la Constitución ordena o prohíbe algo, el Tribunal Constitucional disfruta de un margen de deliberación [...]. La única respuesta correcta es que en estos casos, los derechos fundamentales no ofrecen ninguna respuesta correcta ${ }^{11}$.

La fundamentación del derecho relativo del criterio de generalidad remite a los márgenes de posibilidad apuntados, desde los cuales Alexy prepara las objeciones contra el concepto de principio para la teoría de los derechos fundamentales: La invalidez de los principios (i); los principios absolutos (ii); y la amplitud del concepto de principio (iii); este último resulta el más interesante para mi reflexión pues critica el hecho de que los principios puedan referirse bien a derechos individuales como a bienes colectivos y permite enlazar así con la diferencia entre principios y valores (Alexy, 1993:125) ${ }^{12}$.

En el momento en que respecto a los principios y los valores se intercalan los adjetivos fundamentales y humanos para los derechos, resulta de ayuda recordar el origen de resistencia del mundo jurídico (Villaverde et alt., 2004:17) $)^{13}$ y no obviar la transformación de los Derechos Humanos en Derechos Fundamentales cuando se positivizan; gracias al derecho previamente positivado en aras de proteger y promover la dignidad humana, no sin controversias entre modelos:

«La idea básica del hombre sujeto y no objeto, del ser humano que por el hecho de nacer es portador de derechos inalienables e inviolables, se sintetiza en el concepto de dignidad humana, como valor consustancial de la persona [sin embargo] no cabe identificar sin más derechos humanos y derechos fundamentales, porque el iusracionalismo y el positivismo, aun pudiendo coincidir en una idea común (el individuo y su dignidad), son modelos que se construyen de manera muy diferente.

\section{Ibídem LVI}

12 Alexy, Robert. Teoría de los derechos... p. 125 y ss.

13 «El estudio de los derechos fundamentales tiene como referencia ineludible los derechos humanos, aunque conviene dejar claro que unos y otros no son exactamente lo mismo. Desde siempre el ser humano ha buscado la manera de resistirse al poder de dominación y a lo largo de la historia ha ido creando fórmulas filosófico-jurídicas para frenar ese poder, [s]in embargo, la idea de derechos humanos tal cual la conocemos hoy es relativamente reciente y se ubica en el pensamiento liberal revolucionario de finales del siglo XVIII», Villaverde, Ignacio; Requejo, Paloma; Aláez, Benito; Fernández, Ignacio; Bastida, Fco. José; Presno, Miguel A. Teoría general de los derechos fundamentales en la Constitución española de 1978. Madrid, Tecnos, 2004, p. 17. Pueden verse también: Ansuategui Roig, Francisco J. «La Historia de los Derechos Humanos» en Soriano, Ramón L. (dir.). Diccionario Crítico de los Derechos Humanos. Universidad Internacional de Andalucía, Sede Iberoamericana, La Rábida, 2000, págs. 71-78 
Puede decirse en una primera aproximación y en términos harto generales que el modelo positivista transforma los derechos humanos en derechos fundamentales. Los incorpora como un elemento esencial del sistema jurídico, que los reconoce y garantiza con la fuerza irresistible del único derecho válido, el derecho positivo, es decir, los respalda con el uso lícito de la fuerza física que ostenta en monopolio el Estado» ${ }^{14}$.

La positivización del Derecho se dibuja como una solución que deja atrás al historicismo y su idea del cambio sin quiebra, los ordenamientos jurídicos pre-estatales, el fundamento de los derechos en normas históricas cuya validez residía en la antigüedad y se nutría de la alusión a las Leyes Fundamentales y a la Constitución Histórica, y da paso a otros modelos. El modelo iusracionalista (iusnaturalista) donde el Derecho Natural (la decisión) confiere la validez a las normas, el fundamento ya no es pues la Historia, se pasa del estado de naturaleza al pacto social donde el Estado es garante de los derechos gracias a la ley que dispone cuando se sobrepasan los límites de los derechos y donde el juez determina la infracción y la sanción... en una especie de continuo en el que el reclamo de los derechos de participación y las exigencias de la titularidad de estos derechos presentan claras deficiencias del modelo mismo (Villaverde et alt., 2004: 21-24).

De esta manera irrumpen el positivismo y las Constituciones. Los derechos traen causa en la decisión adoptada por el órgano estatal competente. No hay derechos previos ni supraderecho, sólo Derecho positivo producido por órganos estatales que crean y no declaran los derechos; donde el nexo entre derecho y garantía es fundamental gracias al poder público, el cual crea las garantías institucionales, donde se determinan la titularidad y el contenido de los derechos, y qué fuentes concretas crean y regulan la libertad, respeto al contenido de los derechos que sólo se modifican a través del poder constituyente-constituido, donde la Constitución es la norma jurídica suprema (Villaverde et alt., 2004: 24-28).

El derecho positivo en ningún momento dibuja un paisaje de concreción finita, delimitada y mínima, sino que presenta una espacio abierto para la interpretación de la Constitución:

«Se trata más bien de normas que tienen una estructura abierta, de programación finalista, que las convierte en marco jurídico susceptible de diversas concreciones [...]. El campo de actuación de la interpretación

${ }^{14}$ Y continúa: «Reclamar un derecho fundamental no consistirá en apelar sin más al respeto a un derecho natural de la persona. La apelación no tendrá virtualidad ante los poderes públicos si ese derecho no está previamente positivado, o sea, incorporado y garantizado como derecho positivo, único alegable ante los tribunales». Villaverde, Ignacio; Requejo, Paloma; Aláez, Benito; Fernández, Ignacio; Bastida, Fco. José; Presno, Miguel A. Teoría general de los derechos fundamentales... p.18 
constitucional no son los resquicios, sino el propio marco constitucional. Además, la apertura constitucional entraña una apertura cognitiva del sistema jurídico hacia la realidad que es objeto de su formación, lo cual es particularmente relevante en materia de derechos fundamentales. Hay una constante relación de conocimiento del sistema jurídico con el medio social, porque es esencial para su eficacia saber concretar cuáles son las expectativas sociales que quedan garantizadas en los enunciados iusfundamentales. El contexto social forma parte de la teoría de la Constitución (y de los derechos fundamentales) que se encierra en el texto constitucional. Labor del intérprete es aprehender esa teoría para así poder hacer una interpretación constitucionalmente adecuada no sólo de los enunciados iusfundamentales, sino también de la realidad (contexto) que abstractamente está regulada en ellos» (Villaverde et alt., 2004: 57).

En un espacio como el descrito, la concreción de las normas constitucionales integrarán la creación y la aplicación del derecho. Parlamento y Tribunal Constitucional deberán legitimar las lógicas de sus actuaciones en un encuentro entre la legitimidad política de las urnas y la legitimidad jurídica garante frente a los arbitrios y las extralimitaciones.

Estos espacios de competencia y legitimidad tienen interés en tanto que se relacionan con el desarrollo de la idea de dignidad humana. Coincido con los postulados de Barranco Avilés cuando afirma que la reflexión en torno a la figura los derechos fundamentales lleva a distinguir tres vertientes -la ética, la política y la jurídica- y reitera que «la función política es factible por el enlace ético de los derechos. Los derechos fundamentales sirven de legitimidad al poder sólo si aparecen como elementos de protección del individuo o como cauces de la integración de éste» (Barranco, 2000:113) ${ }^{15}$. Pero hay diversidad entre las teorías del modelo republicano y del modelo liberal sobre cómo el individuo se integra en sociedad, sobre cómo la persona se inserta en el Estado. Nuestra cultura jurídica es fundamentalmente de tradición liberal, en la cual la capacidad de elección reside en el individuo y no en el grupo, y se entiende la libertad de elección como paso previo a la idea superior de autonomía.

La realidad, donde las exigencias éticas de dignidad del individuo cuya satisfacción es condición de legitimidad para el poder político, está repleta de significados para la idea de individualismo. Barranco presenta entre otros individualismos, el abstracto, el político, el económico, el metodológico, el ontológico, o el normativo, y afirma que «[p]uesto que la crítica al individualismo

${ }^{15}$ Barranco Avilés, M. del Carmen. La teoría jurídica de los derechos fundamentales. Madrid, Dykinson, 2000, p. 113. Véase también: Sarmiento, Daniel. «Who’s Afraid of the Charter; The Court of Justice, National Courts and the New Framework of Fundamental Rights Protection in Europe», Common Market Law Review. 2013, 50, págs. 1267-1304 
liberal se traduce en crítica a la idea de los derechos -que ciertamente se vincula a esta tradición no sólo histórica, también conceptualmente- es preciso distinguir las distintas funciones que puede desempeñar la categoría en el lenguaje político» (Barranco, 2000:117) ${ }^{16}$. En paralelo, Barranco hace una llamada de atención a las condiciones reales de existencia del individuo, del ser humano titular de derechos, que se enlazaría con la idea de fundamento y sus esquemas de aproximación a los derechos fundamentales en su función subjetiva (Barranco, 2000: 312).

Desde la división entre las teorías de los derechos morales y las teorías de los valores, Barranco pone de manifiesto que lo determinante es el fundamento en la medida en que condiciona la forma en la que se interpreta el alcance subjetivo de los derechos positivos (Barranco, 2000: 312).

La vinculación entre sujeto y derecho protegido varía según nos instalemos en las teorías de los derechos morales o en las de los valores pues, como afirma la autora, los defensores de estas últimas se orientan en la línea de la crítica al individualismo abstracto que se encontraba en el origen del reconocimiento histórico de los derechos (Barranco, 2000: 314).

Otra crítica orientada a la importancia del enraizamiento de los derechos, y que sirve para proponer una pausa a esta reflexión, es la ofrecida por Anna Pintore en relación a los derechos insaciables que, tal y como entiende, encarnan las teorías de Ferrajoli, especialmente desde Derecho y razón (Pintore, 2001: 243) ${ }^{17}$.

Pintore dibuja un panorama en el que los derechos han quedado abandonados a sí mismos o a la frágil Historia del Constitucionalismo, y se pregunta cómo fundamentar los derechos, qué derechos fundamentar y cómo insertar en la Historia, en la realidad, los derechos ya fundamentados (Pintore, 2001: 244).

Se reconoce la necesidad de las Auctoritas puesto que no hay teorías (auto)suficientes, y sigue a Bobbio -protección de los derechos, más allá de la justificación- desde el lado más tratable de la administración. Pintore piensa que Ferrajoli sacrifica a la democracia misma al minusvalorar el problema formal de la gestión del contenido sustancial de los derechos y al sesgar el vínculo entre derecho y titular del mismo:

«Definir como «democracia sustancial» al estado de derecho, equivale, de hecho, a otorgar una solución semántica a un problema normativo. Equivale a resolver in limine y de forma oculta con una operación de limpieza lingüística, precisamente, el problema crucial de la justificación filo-

16 Barranco Avilés, M. del Carmen. La teoría jurídica ... p. 117

17 Pintore, Anna. «Derechos insaciables», en Ferrajoli, Luigi. Los fundamentos de los derechos fundamentales. Madrid, Trotta, 2001, págs: 243-265; Ferrajoli, Luigi. Teoría del garantismo penal. Prólogo de N. Bobbio, Trotta 10ª ed., Madrid, 2011, 1024 p. 
sófica de las democracias constitucionales: el problema, justamente, de cómo conciliar el estado de derecho (contenidos) con la democracia (forma), de cómo resolver en un equilibrio aceptable la tensión entre derechos fundamentales y el principio del autogobierno. [...] Añadiré, finalmente, que de la definición del estado de derecho como democracia (sustancial) aflora una visión teórica de la democracia constitucional, en el fondo,

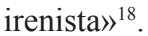

Desde estas afirmaciones se dará continuidad a la tarea ardua, pero no imposible de demostrar la posibilidad de una existencia armónica entre democracia y derechos. La compleja conciliación planteada por Pintore entre contenidos y forma, entre estado de derecho y democracia, interpela al estudio interdisciplinar de los derechos fundamentales y del principio del autogobierno, a la vez que plantea futuros interrogantes en relación a la democracia constitucional actual.

\section{BIBLIOGRAFÍA}

Alexy, R. Teoría de los derechos fundamentales. Madrid, Centro de Estudios Constitucionales, 1993, $607 \mathrm{p}$.

— «Constitutional Rights, Balancing, and Rationality», en Ratio Juris. Vol. 16 N. 2 Junio 2003, págs. 131-40

— Law and Correctness, en Legal Theory at the End of the Millennium. Ed. M. D. A. Freeman, Oxford: Oxford University Press. 1998, págs. 205-221

Ansuategui Roig, F.J. «La Historia de los Derechos Humanos» en Soriano, Ramón L. (dir.). Diccionario Crítico de los Derechos Humanos. Universidad Internacional de Andalucía, Sede Iberoamericana, La Rábida, 2000, págs. 71-78

BARRANCO Avilés, M. del C. La teoría jurídica de los derechos fundamentales. Madrid, Dykinson, 2000, $444 \mathrm{p}$.

CoBos, P.; JARA, M.; FioL, B. (Dir.) «Plasmación jurídica de la dignidad de la persona», en VV. AA. Progreso humano, derechos humanos: XXXI Congreso Universitario Internacional UNIV'98, Sevilla, Colegio Mayor Almonte, 1998, 451 p.

Díez-Picazo, L.M. Sistema de Derechos Fundamentales, Madrid, S.L. Civitas Ediciones $2003,510 \mathrm{p}$.

FERnÁndeZ, F. «La Teoría jurídica de los derechos fundamentales en la doctrina constitucional», en Revista Española de Derecho Constitucional, Año 13. Núm. 39. Septiembre-Diciembre 1993 pp. 195-247

Ferrajoli, L. Teoría del garantismo penal. Prólogo de N. Bobbio, Trotta $10^{\mathrm{a}}$ ed., Madrid, 2011, $1024 \mathrm{p}$.

— Los fundamentos de los derechos fundamentales. Madrid, Trotta, 2001, $392 \mathrm{p}$.

Ferrater Mora, José. «Derecho», en Diccionario de filosofía. Tomo I. (A-D), Barcelona, Ariel Filosofía, 2009. pp: 817-819

${ }_{18}$ Pintore, Anna. «Derechos insaciables... » p. 250 
— «Fundamento», en Diccionario de filosofía. Tomo II. (E-J), Barcelona, Ariel Filosofía, 2009. pp: 1413-1414

Fioravant, M. Constitucionalismo. Experiencias históricas y tendencias actuales. Madrid, Trotta 2014. 167 p.

Garcia Amado, J. A. Teorías del sistema jurídico y concepto de derecho. Anuario de Filosofía del Derecho (2), 1985, págs. 297316.

Greco, M. «The Politics of Indeterminacy and the Right to Health», en Theory, Culture and Society, 2006, 21. 6, págs: 1-22

MASSINI CORREAS, Carlos Ignacio. «La interpretación jurídica como interpretación práctica», en Persona y derechos: Revista de fundamentación de las Instituciones Jurídicas y de Derechos Humanos, N. 52, 20015, págs: 413-444

Pintore, Anna. «Derechos insaciables», en FERrajoli, Luigi. Los fundamentos de los derechos fundamentales. Madrid, Trotta, 2001, pp: 243-265

- El Derecho sin verdad. Madrid. Dykinson 2006, 234 p.

SANTAYAnA, George. La vida de la razón o Fases del progreso humano. Adaptación, introducción, notas y anexos de José Beltrán Llavador; trad. de Aida A. De Kogan, Madrid, Tecnos, 2005, 314 p.

Schlag, Pierre. «The dedifferentiation problem», en Continental Philosophy Review, 200942 (1). Págs.: 35-62.

SARmiento, Daniel. «Who's Afraid of the Charter; The Court of Justice, National Courts and the New Framework of Fundamental Rights Protection in Europe», Common Market Law Review. 2013, 50, págs. 1267-1304

Villaverde, Ignacio; ReQueJo, Paloma; AlÁez, Benito; Fernández, Ignacio; BasTIDA, Fco. José; PRESNO, Miguel A. Teoría general de los derechos fundamentales en la Constitución española de 1978. Madrid, Tecnos, 2004, 235 p.

— «Verdad y Constitución. Una incipiente dogmática de las ficciones constitucionales», en Revista española de derecho constitucional. Año N. 36, Núm. 106, 2016, págs: 149-201.

Este trabajo fue presentado en forma de comunicación en el Congreso sobre «Justicia, Administración Pública y Derechos Fundamentales» celebrado los días 16 y 17 de noviembre de 2017, en la Facultad de Derecho y CC. EE. y Empresariales de la Universidad de Córdoba.

La comunicación se presentó en el Bloque I. Cuestiones actuales de Teoría General de los Derechos Fundamentales.

El congreso se enmarcó en el Proyecto de Investigación DER201567695-C2-2-P (MINECO/FEDER). 


\title{
DERECHOS FUNDAMENTALES: INDETERMINACIÓN CONCEPTUAL, PLASMACIÓN JURÍDICA E INTERPRETACIÓN
}

\author{
Fundamental Rights: conceptual indeterminacy, \\ legal embodiment and interpretation
}

\author{
Estela Camus García \\ Universidad Loyola Andalucía (España) \\ escamus@uloyola.es
}

http://dx.doi.org/10.18543/ed-66(2)-2018pp105-115

\section{Copyright}

Estudios de Deusto es una revista de acceso abierto, lo que significa que es de libre acceso en su integridad. Se permite su lectura, la búsqueda, descarga, distribución y reutilización legal en cualquier tipo de soporte sólo para fines no comerciales, sin la previa autorización del editor o el autor, siempre que la obra original sea debidamente citada y cualquier cambio en el original esté claramente indicado 